\title{
Precipitation Impacts Dissemination of Three Sooty Blotch and Flyspeck Taxa on Apple Fruit
}

\author{
Hafizi Rosli, ${ }^{1}$ Jean C. Batzer, ${ }^{2}$ Edward Hernández, ${ }^{2}$ Gustavo Beruski, ${ }^{3}$ Philip M. Dixon, ${ }^{4}$ and Mark L. Gleason ${ }^{2, \dagger}$ \\ ${ }^{1}$ School of Biological Sciences, Universiti Sains Malaysia, Penang, Malaysia \\ ${ }^{2}$ Department of Plant Pathology and Microbiology, Iowa State University, Ames, IA, U.S.A. \\ ${ }^{3}$ Department of Biosystems Engineering, ESALQ, University of São Paulo, Piracicaba, Brazil \\ ${ }^{4}$ Department of Statistics, Iowa State University, Ames, IA, U.S.A.
}

\begin{abstract}
The spatial dissemination of three prevalent taxa of sooty blotch and flyspeck (SBFS) fungi under several levels of precipitation was compared during 2015 and 2016 in an Iowa apple orchard. Overhead irrigation was used to supplement ambient precipitation in order to insure SBFS spore dissemination and colony development. There were five irrigation levels, involving 1-min-long periods of irrigation that were imposed either once or twice per hour at intervals of 3,6, or $12 \mathrm{~h}$, as well as a nonirrigated control. Preselected apple fruit were inoculated with one of the three SBFS taxa to serve as sources of inoculum. Dissemination from these inoculated apple fruit was assessed at harvest by counting SBFS colonies on watersprayed and nontreated fruit. As a further control, additional fruit were enclosed in fruit bags throughout the fruit development period. In both 2015 and 2016, the number of colonies of the SBFS fungus Peltaster gem-

the linear relationship between the duration of irrigation-imposed precipitation levels and the number of colonies on the water-sprayed apple fruit was similar for $P$. gemmifer (slope $=0.09$ ), Stomiopeltis sp. RS1 (slope $=0.07$ ), and Microcyclosporella mali (slope $=0.13$ ); whereas, in 2016, the slope was higher for P. gemmifer (0.28) than for Stomiopeltis sp. RS1 (-0.09) or M. mali (0.06). The results indicated that dissemination of $P$. gemmifer increased sharply in response to increased irrigationimposed precipitation, and that dissemination patterns differed considerably among the three SBFS taxa. The apparent advantage of $P$. gemmifer in precipitation-triggered dissemination may stem from its ability to produce spores rapidly by budding. To our knowledge, this is the first article to assess splash dispersal by SBFS fungi in the field and the first to document taxon-specific patterns of dissemination in this pathogen complex.
\end{abstract} mifer per apple increased sharply as the duration of irrigation increased, whereas the number of colonies of Microcyclosporella mali increased to a lesser extent and Stomiopeltis sp. RS1 showed no increase. In 2015,
Keywords: Dissemination, field dispersal, irrigation, leaf wetness duration, SBFS
Sooty blotch and flyspeck (SBFS) is a fungal disease complex of apple and other fruit that occurs in moist growing regions worldwide, producing superficial dark smudges or clusters of dots that can render fruit unsuitable for fresh-market sale (Gleason et al. 2011, 2019; Williamson and Sutton 2000). In Iowa and elsewhere in the eastern half of the United States, most apple growers manage SBFS by applying fungicide sprays every 1 to 2 weeks throughout the fruit development period (Gleason et al. 2011). This approach can lead to unnecessary sprays because it does not account for fluctuations in disease risk due to environmental conditions, especially the duration of periods of wetness or high relative humidity (Brown and Sutton 1995; Duttweiler et al. 2008). Therefore, use of weatherbased warning systems to time fungicide sprays has been proposed in order to reduce input costs and minimize unnecessary fungicide spraying (Duttweiler et al. 2008; Rosli et al. 2017).

The SBFS complex is among the most diverse plant pathogen assemblages ever documented, with at least 60 named or putative

${ }^{\dagger}$ Corresponding author: M. L. Gleason; mgleason@ iastate.edu

Funding: H. Rosli was awarded an Academic Staff Training Scheme scholarship by Universiti Sains Malaysia and Ministry of Higher Education Malaysia for the Ph.D. program at Iowa State University.

*The $\boldsymbol{e}$-Xtra logo stands for "electronic extra" and indicates that supplementary materials are published online.

The author(s) declare no conflict of interest.

Accepted for publication 18 March 2020.

(C) 2020 The American Phytopathological Society species in the United States alone (Gleason et al. 2011) and more than 100 worldwide (Gao et al. 2015; Gleason et al. 2019; Medjedović et al. 2014; Zhao et al. 2016). Recognition that SBFS comprises multispecies complexes within individual apple orchards and that SBFS species assemblages varied among geographic regions triggered exploration of physiological and ecological differences among SBFS species in order to improve effectiveness of disease management (Batzer et al. 2010, 2015; Díaz Arias et al. 2010; Johnson and Sutton 2000; Ismail et al. 2016; Tarnowski et al. 2003). However, little is known about taxon-specific patterns of dissemination within orchards or among apple fruit on the same tree, although these patterns could influence the risk of epidemic spread (Gleason et al. 2011, 2019).

Preliminary evidence from field trials in Iowa apple orchards suggested that SBFS species vary widely in their mode of spore production and means of dissemination. For example, Peltaster spp. produce single-celled blastospores via budding, a process similar to spore production by yeast that enables multiple infection cycles per season (Batzer et al. 2012; Johnson et al. 1996, 1997; Williamson and Sutton 2000). Likewise, Microcyclosporella mali produces multicelled scolecosporous (slender thread-like) conidia by microcyclic conidiation, and is a dominant member of the SBFS complex in eastern Europe and the eastern United States (Batzer et al. 2016; Frank et al. 2010). In contrast, Stomiopeltis spp. have not been observed to produce asexual conidia on apple fruit or in culture but do induce infections, presumably from ascospores, in Iowa (Ismail et al. 2016). Ascospores of Stomiopeltis spp. have been observed on overwintered fruit in North Carolina (T. B. Sutton, personal communication) and Iowa (J. C. Batzer, unpublished data). The idea that SBFS taxa differ considerably in dissemination dynamics gained support from a recent study to determine timing of natural inoculation in an Iowa orchard. During 5 years of observation at six locations in Iowa, the majority of early-season SBFS 
infections were caused by Stomiopeltis spp. (Batzer et al. 2012; Ismail et al. 2016). However, during an exceptionally rainy growing season, Peltaster spp. showed a marked increase in number of colonies on fruit as the season progressed, in contrast to other prevalent SBFS taxa for which colonies either remained constant or decreased (Ismail et al. 2016).

Based on these preliminary findings, we hypothesized that rainfall periods differentially impact dissemination of SBFS taxa. Our objective was to assess whether precipitation differentially affects the dissemination of three predominant SBFS taxa (Peltaster gemmifer, Stomiopeltis sp. RS1, and Microcyclosporella mali) among fruit within the same apple tree.

\section{Materials and Methods}

Field site. Trials were conducted during 2015 and 2016 at the Iowa State University Horticulture Research Station (ISUHRS; $42^{\circ} 06^{\prime}$ $23.8^{\prime \prime} \mathrm{N}, 93^{\circ} 35^{\prime} 23.3^{\prime \prime} \mathrm{W}$ ) in a 0.32 -ha apple orchard that was planted in 2003. Spacing within and between rows was 2.5 and $4.4 \mathrm{~m}$, respectively (Supplementary Fig. S1). Each subplot consisted of a row segment of six adjacent trees of cultivar Golden Delicious. Golden Delicious was selected because its light-yellow color at fruit maturity facilitates visual detection of SBFS colonies (Williamson and Sutton 2000). For each end of each subplot of the Golden Delicious trees, a single tree of cultivar Gala acted as a guard tree. These trees were not included in the experiment. To control apple scab (Venturia inaequalis) and rusts (Gymnosporangium spp.), a tank mix of the fungicides mycobutanil (Rally 40 WSP) and fenarimol (Rubigan), which have relatively low activity against SBFS fungi (Ismail et al. 2016), was applied from green tip to petal fall. The fungicides thiophanatemethyl (Topsin M, $1.05 \mathrm{~kg} / \mathrm{ha}$ ) plus captan (Captan 50W, $6.73 \mathrm{~kg} / \mathrm{ha}$ ) were sprayed after petal fall on 27 May 2015 and 29 May 2016 to eliminate SBFS infections from ambient inoculum sources prior to artificial inoculations. Irrigation treatments were assigned to rows, and inoculation treatments were assigned to subplots within rows (Supplementary Fig. S1).

Irrigation treatments and leaf wetness monitoring. A computer-controlled overhead irrigation system (H. Rosli, unpublished data) was established to apply different precipitation treatments, with each row receiving a single irrigation treatment. The irrigation treatments were not replicated, because the focus of the experiment was to compare dissemination patterns among three SBFS taxa as a result of precipitation rather than to quantify responses of each taxon to different levels of irrigation intensity. Thus, the primary purpose of the irrigation treatments was to provide sufficient precipitation to facilitate dissemination of inoculum. Irrigation nozzles (Pop-up Spray Head Sprinklers, model 1812AP; Rain Bird, Azusa, CA, U.S.A.) were placed at an approximately 4-m height at the top center of the tree canopy in four contiguous rows of trees. Subplots located at the north end of each row had no overhead irrigation system (ambient precipitation only). Three adjacent trees in the middle of each subplot were used for data acquisition. Five irrigation treatments were imposed: once per hour for $12 \mathrm{~h}$ (8 P.M. to 8 A.M.); once per hour for 6 h ( 2 A.M. to 8 A.M.); twice per hour for 6 h ( 2 A.M. to 8 A.M.); twice per hour for 3 h (5 A.M. to 8 A.M.); and no irrigation (ambient precipitation only) (Table 1; Supplementary Fig. S1). Each irrigation event lasted for $1 \mathrm{~min}$. The irrigation regimes were assigned arbitrarily to tree rows in both 2015 and 2016, whereas the nonirrigated control treatment remained in the same location in both years. The irrigation treatments started on the date of inoculation and ended on the date of harvest in both 2015 and 2016 (Table 2).

Flat, printed-circuit leaf wetness sensors (model 237-L; Campbell Scientific, Logan, UT) whose surfaces had been coated with latex paint to increase their sensitivity to moisture (Gillespie and Kidd 1978) were used to estimate leaf wetness duration (LWD) and verify proper operation of the irrigation heads. Three sensors were placed in each of two adjacent subplots in each irrigation treatment: one sensor in the top third of the tree canopy, one in the center of the canopy, and one in the lower third. All wetness sensors in the tree canopy were mounted on the end of a section of PVC pipe, facing north at an inclination of $45^{\circ}$ to horizontal. Data loggers (model CR10; Campbell Scientific) recorded wetness sensor data at 15-min intervals. Daily and cumulative rainfall at the ISUHRS from date of inoculation to date of harvest was obtained from a tipping-bucket rain gauge (model TE525-L; Campbell Scientific) by an automated datalogger (model CR10; Campbell Scientific) located within $1,000 \mathrm{~m}$ of the study plot (https://www.mesonet.agron. iastate.edu/).

Table 2. Chronology and key dates from first-cover fungicide spray until harvest

\begin{tabular}{lll}
\hline & \multicolumn{2}{c}{ Year } \\
\cline { 2 - 3 } Category and input & \multicolumn{1}{c}{$\mathbf{2 0 1 5}$} & \multicolumn{1}{c}{$\mathbf{2 0 1 6}$} \\
\hline First-cover fungicide spray applied $^{\mathrm{w}}$ & 27 May & 29 May \\
Apple fruit bagged $^{\mathrm{x}}$ & 16 June & 31 May \\
Inoculation treatment imposed $^{\mathrm{y}}$ & 8 July & 20 June \\
Harvest $^{\mathrm{z}}$ & 22 September & 18 September \\
\hline
\end{tabular}

${ }^{w}$ Applied to all trees in the experiment plot.

${ }^{x}$ Fifty apple fruit per tree in each of three trees in the center of each six-tree subplot were bagged from shortly after the first-cover fungicide spray until inoculation, for a total of 150 fruit per subplot.

y Ten apple fruit per tree were inoculated with one of the three sooty blotch and flyspeck (SBFS) taxa (Peltaster gemmifer, Stomiopeltis sp. RS1, or Microcyclosporella mali). Fifteen additional fruit per tree were sprayed with water at the time of inoculation, and five fruit per tree were enclosed in fruit bags throughout the fruit development period. To minimize infection by ambient SBFS inoculum, all of the apple fruit designated for inoculation treatments were covered with fruit bags for 20 to 22 days prior to the day of inoculation. Bags covering the apple on each tree were removed during the inoculation; then, the fruit were rebagged until the following day to prevent inoculum wash off. In each year, all inoculation treatments were applied on the same day.

${ }^{\mathrm{z}}$ Fifty apple fruit per tree from the inoculation treatments were collected in separated bags according to the colored flags. An additional 30 nontreated fruit from the same trees that had never been bagged were collected to supplement data from the treated fruit.

Table 1. Irrigation treatments in an apple field plot at Iowa State University Horticulture Research Station in 2015 and 2016

\begin{tabular}{lcccc}
\hline & \multicolumn{3}{c}{ Irrigation treatment } \\
\cline { 2 - 5 } Treatment details & $\mathbf{1}$ & $\mathbf{2}$ & $\mathbf{3}$ & $\mathbf{4}$ \\
\hline Hours per irrigation period & $5-8$ A.M. & $2-8$ A.M. & $2-8$ A.M. & 8 p.m.-8 A.M. \\
$\begin{array}{l}\text { Total irrigation hours } \\
\begin{array}{l}\text { Frequency of water delivery per irrigation } \\
\text { period }\end{array}\end{array}$ & 3 & 6 & 6 & 12 \\
$\begin{array}{c}\text { Total number of water delivery intervals per } \\
\text { irrigation period }\end{array}$ & 30 & 60 & 30 & 60 \\
\hline
\end{tabular}

\footnotetext{
${ }^{x}$ No irrigation treatment. Naturally occurring rain and dew.

y Overhead irrigation was applied every 30 or 60 min within the period of irrigation.

${ }^{\mathrm{z}}$ Each irrigation spray interval lasted for $1 \mathrm{~min}$ in all irrigation treatments.
} 
Inoculation treatments. All trees in the trial were inoculated in the same manner. Within 14 days after petal fall but before the first-cover fungicide spray was applied, 50 fruit clusters per tree on each of 16 trees were thinned manually to one fruit per cluster. These fruit were arbitrarily assigned to one of five inoculation treatments (Table 2). Ten apple fruit per tree were inoculated with one of the three SBFS taxa from Mark Gleason's collection at Iowa State University: P. gemmifer (isolate CUE2b), Stomiopeltis sp. RS1 (isolate PEC6a), or M. mali (isolate MWD7a). For each SBFS fungus, 160 fruit were inoculated each year. Fifteen additional fruit per tree, designated water-sprayed controls, were sprayed with sterile distilled water (SDW) at the time of inoculation; these fruit totaled 240 fruit per year. Five apple fruit per tree were designated bagged controls and enclosed in fruit bags (Red Apple Bag number 6 large; Wilson Orchard \& Vineyard Supply; (https://www.wilsonirr.com/ecommerce/red-apple-bag-6-large.php) throughout the fruit development period, totaling 80 fruit per year. At harvest, 30 additional fruit from each tree that had received none of these treatments were also sampled as an additional control treatment (nontreated, nonbagged control), including 1,440 fruit per year (Table 2). All of the apple fruit designated for inoculation treatments, including the water controls and bagged controls, were covered with fruit bags for 20 to 22 days prior to the day of inoculation to minimize infection by ambient SBFS inoculum. Bags covering the apple on each tree were removed during the inoculation; then, the fruit were rebagged until the following day to prevent inoculum wash off. Thus, inoculated apple fruit were the main sources of inoculum, water-sprayed fruit were used to assess secondary spread in the field, fruit that were never covered were used to represent the combination of artificially inoculated and natural sources (SBFS spores that landed on fruit after the fungicide sprays), and fruit that were bagged for the entire season were used to gauge the efficacy of the bags in preventing inoculum from reaching these fruit.

Inoculum preparation and application. Two-week-old cultures of $P$. gemmifer, Stomiopeltis sp. RS1, and M. mali, grown under intermittent ambient light at room temperature $\left(24\right.$ to $\left.26^{\circ} \mathrm{C}\right)$ on potato dextrose agar (PDA), were used to prepare inoculum suspensions. The surface of colonies on five to six PDA plates of each SBFS taxon were scraped using a metal spatula, transferred to a blender, homogenized with $300 \mathrm{ml}$ of SDW, and filtered through three layers of sterile cheesecloth. The suspensions, consisting of spores and mycelial fragments, were adjusted to $10^{8} \mathrm{ml}^{-1}$ using a hemocytometer and placed in 1-liter spray bottles. All inoculum suspensions were prepared $12 \mathrm{~h}$ before application and were stored at $5^{\circ} \mathrm{C}$ before being used in the field. Inoculation was carried out according to the assigned inoculation treatment (Table 2). After fruit bags were removed from the designated apple fruit, they were sprayed twice, once for each hemisphere of the apple, until runoff with the designated fungal inoculum. A tray was placed under the apple during the inoculation process to collect any inoculum runoff. The fruit were then recovered immediately with the same bag. Sterile distilled water was sprayed on the water-sprayed fruit. The bags remained on all sprayed fruit overnight to prevent dripping of inoculum or water onto other fruit. The next day, all bags were removed except for the five fruit per tree that remained bagged all season. At harvest, these fruit bags were removed and fruit were sorted into separate plastic bags according to treatment, then stored at $2{ }^{\circ} \mathrm{C}$ until evaluation.

SBFS colony characterization. At harvest, the number of SBFS colonies displaying the morphologically distinct mycelial types associated with P. gemmifer (punctate), Stomiopeltis sp. RS1, (ramose), and M. mali (ridged honeycomb) (Batzer et al. 2005) were counted on each water-sprayed apple under a dissecting microscope. The percentage of surface area covered by each species was estimated for inoculated apple fruit.

To verify the identity of SBFS taxa that had been provisionally identified on water-sprayed apple fruit according to mycelial type, DNA was extracted from mycelium of five arbitrarily selected colonies of each mycelial type (Duttweiler et al. 2008).
After $10 \mu \mathrm{l}$ of PrepMan Ultra Sample Preparation Reagent (Applied Biosystems, Foster City, CA, U.S.A.) was placed on a colony of each mycelial type, the colony was scraped from the apple peel using the pipette tip and suspended in the droplet; the suspension was then mixed with $40 \mu$ l of PrepMan reagent and fungal DNA was extracted following manufacturer instructions. The protocol of Duttweiler et al. (2008) was followed to amplify the partial ribosomal DNA (rDNA) using primers ITS-1F (5'-CTTGGTCATTTAGAGGAAGTAA-3') and Myc1-R (5'-ACTCGTCGAAGGAGCTACG-3'). The 50- $\mu \mathrm{l}$ PCR mixture contained $4 \mathrm{mM} \mathrm{MgCl2}, 10 \mu \mathrm{l}$ of $10 \times$ Promega buffer, $0.2 \mathrm{mM}$ dNTPs, $0.75 \mu \mathrm{M}$ each primer, $1.25 \mathrm{U}$ of Taq polymerase (Promega Corp., Madison, WI, U.S.A.), and $2 \mu \mathrm{l}$ of DNA template. The PCR thermal cycler conditions were as follows: initial denaturation at $95^{\circ} \mathrm{C}$ for $95 \mathrm{~s}, 35$ cycles of denaturation at $95^{\circ} \mathrm{C}$ for $60 \mathrm{~s}$ and annealing at $58^{\circ} \mathrm{C}$ for $60 \mathrm{~s}$, and extension at $72^{\circ} \mathrm{C}$ for $5 \mathrm{~min}$. The PCR product was purified with Illustra GFX PCR DNA and Gel Band Purification Kit (GE Healthcare Bio-Sciences, Pittsburgh, PA, U.S.A.), and sequenced with the primers ITS-1F and Myc1-R at the DNA Sequencing and Synthesis Facility at Iowa State University, using an Applied Biosystems DNA Analyzer (model 3730xl; Life Technologies, Carlsbad, CA, U.S.A.). Sequences were edited manually using BioEdit (Hall 1999) before BLAST searches were performed to compare with sequences in the GenBank database (National Center for Biotechnical Information, Bethesda, MD, U.S.A.).

Statistical analysis. Due to unequal variation in SBFS colony counts on both water-sprayed and nontreated apple fruit, these data were log-transformed (natural $\log$ ) prior to analysis. PROC MIXED (SAS Inc., Durham, NC, U.S.A.) was used with replicate, hour, event, isolate, isolate-hour, and isolate-event as the fixed effects. The log-transformed counts for the three SBFS taxa were considered repeated measures on the same tree using a compound symmetric structure. Slopes were estimated for counts of $P$. gemmifer, Stomiopeltis sp. RS1, and M. mali with a 95\% confidence interval. Differences in log (number of SBFS colonies) in the nonirrigated control plot were compared by using PROC GLM (SAS Inc.) with species and replicate-tree included as fixed effects. Least squares means was used to assess significance of differences among SBFS taxa.

To assess correlation between precipitation and colony counts for each SBFS taxon, wetness was treated as a continuous variable. Therefore, LWD associated with irrigation treatment was represented as low (associated with the 3-h irrigation regime), medium (associated with two 6-h irrigation regimes), or high (associated with the 12-h irrigation regime) (Table 1); for the purpose of this analysis, the two 6-h irrigation regimes were merged because the impact of irrigation event on colony counts for these two treatments was not significant $(P=0.1$ for 2015, $P=0.4$ for 2016) (H. Rosli, unpublished data).

\section{Results}

At harvest, all inoculated apple fruit developed colony growth consistent with the mycelial types associated with each inoculated species on 25 to $90 \%$ of the fruit surface. These fruit served as the predominant source of inoculum for secondary spread to watersprayed fruit. The total number of water-sprayed fruit was reduced during the growing season due to fruit drop and physical damage (Table 3). The number of colonies of each taxon observed on the remaining water-sprayed fruit at harvest was used to assess effects of the irrigation treatments on secondary spread of these taxa. Fruit that were bagged all season showed a few SBFS colonies in 2015 but none in 2016.

Precipitation impact on SBFS colony counts. The number of colonies of each SBFS taxon on water-sprayed fruit differed in response to the irrigation treatments. $P$. gemmifer colonies greatly outnumbered those of the other two taxa in both years; this difference increased as duration and frequency of irrigation increased (Fig. 1A and B). Similar trends occurred on nontreated apple fruit 
that had not been bagged (Fig. 1C and D), although the number of colonies per apple was higher than for the water-sprayed apple fruit that had been bagged prior to inoculation (Fig. 1A and B). The effect of number of hours of irrigation treatment $(3,6$, or $12 \mathrm{~h}$ ) significantly $(P<0.05)$ affected number of colonies but neither the effect of frequency of irrigation event (once versus twice per hour) nor interaction between irrigation hour and event was significant $(\mathrm{H}$. Rosli, unpublished data).

In 2015, on both water-sprayed and nontreated apple fruit, $M$. mali colony counts were more strongly correlated with the change of

Table 3. Total number of water-sprayed apple fruit collected from each irrigation treatment at harvest in 2015 and 2016

\begin{tabular}{lrrrrr}
\hline & \multicolumn{5}{c}{ Irrigation treatmenty $^{\mathbf{y}}$} \\
\cline { 2 - 6 } Year & $\mathbf{1}$ & $\mathbf{2}$ & $\mathbf{3}$ & $\mathbf{4}$ & $\mathbf{5}^{\mathbf{z}}$ \\
\hline 2015 & 104 & 100 & 113 & 88 & 140 \\
2016 & 88 & 93 & 72 & 97 & 138 \\
\hline
\end{tabular}

y Irrigation regimes for each treatment: $1=$ irrigated twice/hour, 5-8 A.M., $2=$ irrigated once/hour, 2-8 A.M., 3 = irrigated twice/hour, 2-8 A.M., 4 = irrigated once/hour, 8 P.M. -8 A.M., and $5=$ no irrigation.

${ }^{\mathrm{z}}$ Tree count: $n=12$, compared with $n=9$ for other treatments.
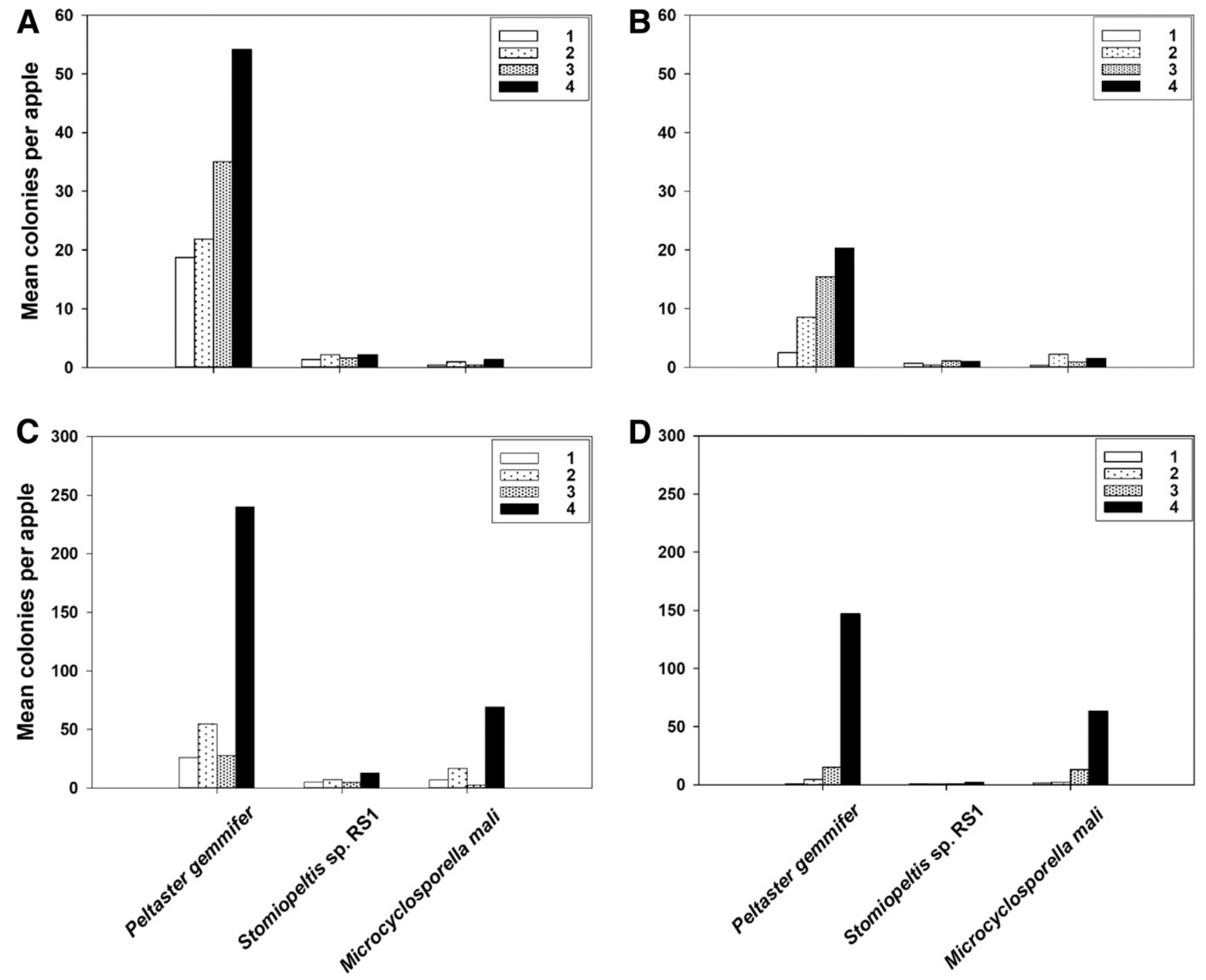

Fig. 1. Mean number of colonies per apple associated with the three most prevalent sooty blotch and flyspeck species in lowa from different irrigation treatments: 1 , irrigated twice/ hour, 5-8 A.M.; 2, irrigated once/hour, 2-8 A.M.; 3, irrigated twice/hour, 2-8 A.M.; and 4, irrigated once/hour, 8 P.M. -8 A.M. A. Water-sprayed, 2015; B, water-sprayed, 2016; C, nontreated, nonbagged control, 2015; and D, nontreated, nonbagged control, 2016. 
Weather data. For the period from inoculation until harvest, mean cumulative LWD for all irrigation treatments was similar between years, whereas cumulative rainfall from date of inoculation to date of harvest was somewhat lower in 2015 (394.5 $\mathrm{mm})$ than in 2016 (503.9 mm) (Table 5). During 2015, however, ambient daily rainfall and dew (based on hourly sensor data from 10 P.M. to 9 A.M.) was higher during 3 weeks immediately following inoculation than in 2016 (Fig. 3). We also evaluated the impact of the irrigation treatments on total LWD during irrigation periods, including dew and rain as well as irrigation. Cumulative LWD ranged from approximately $250 \mathrm{~h}$ in the low irrigation treatment to $700 \mathrm{~h}$ in the high irrigation treatment, and the trend was similar in both years (H. Rosli, unpublished data). The range of cumulative LWD (including natural rain and dew events as well as irrigation) indicated that rain and dew contributed most of the wetness hours.

Verification of SBFS taxa. Sequences obtained from each of five colonies of each mycelial type (punctate, ramose, and ridged honeycomb) were approximately $800 \mathrm{bp}$ in length, and BLAST searches closely matched the sequences deposited in GenBank for two of the inoculated SBFS taxa: the punctate mycelial type showed 98 to $100 \%$ identity to $P$. gemmifer (AY598889, AY598890, and MF075291) and the ridged honeycomb mycelial type showed $100 \%$ similarity to M. mali (KP400560, KC859001, and GU570530). However, the ramose mycelial types from water-sprayed apple fruit showed 99 to $100 \%$ match to different Stomiopeltis sp. R5.1 and RS5.2 (AY160174, FJ438375, and
FJ438376) that were previously isolated from North Carolina, rather than that of the Iowa PEC6a isolate of Stomiopeltis sp. RS1 (AY598882) used for inoculation ( 87 to $89 \%$ base pair identity). This indicated that most of the ramose colonies on the water-sprayed fruit may have been the result of inoculum originating from sources other than the inoculated fruit in the study. Although the inoculated fruit developed infections, these were not the main source of Stomiopeltis sp. inoculum on water-sprayed or nontreated fruit.

\section{Discussion}

To our knowledge, this is the first study of dissemination of SBFS fungi on fruit, the first comparison of SBFS taxa with regard to their patterns of dissemination in the field, and the first to relate these patterns to environmental conditions. In both years, $P$. gemmifer dissemination from inoculated apple fruit to water-sprayed fruit far exceeded that of the other two SBFS taxa; this trend was particularly pronounced for treatments that featured more prolonged or frequent irrigation events (Fig. 1A and B). The same trend was apparent for nontreated fruit that had not been bagged prior to the date of inoculation of other fruit (Fig. 1C and 1D). The results support the hypothesis that the greater ability of Peltaster spp. to spread among fruit is related to their greater ability to produce secondary cycles of inoculum during wet periods, and is consistent with previous field observations (Batzer et al. 2012; Ismail et al. 2016). This ecological distinction adds an important dimension to documented differences among recently discovered SBFS taxa regarding phenology
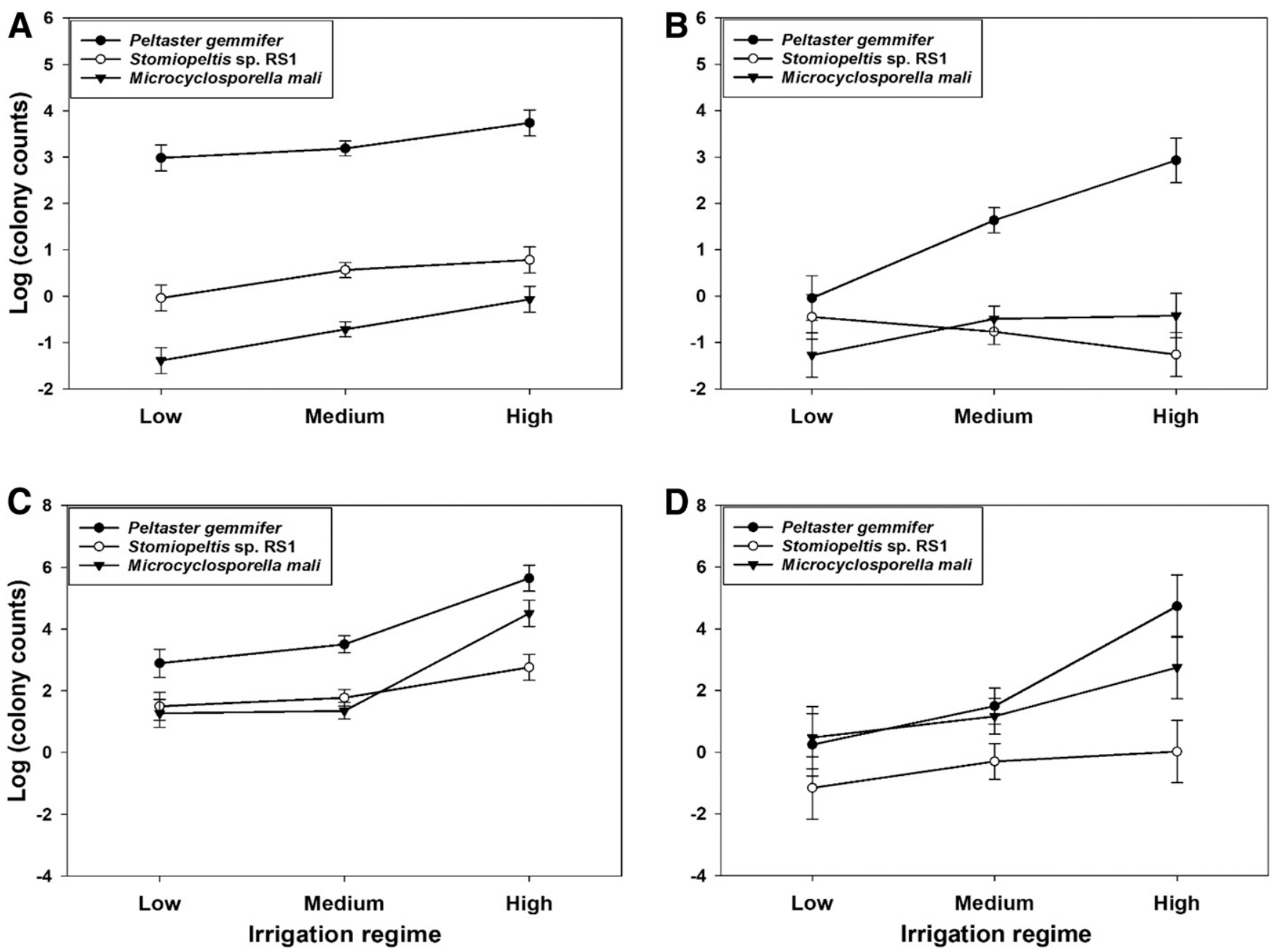

Fig. 2. Effect of irrigation regime on log (colony counts) associated with the three most prevalent sooty blotch and flyspeck species in a central lowa orchard. In this analysis, the two 6-h irrigation regimes were merged together, and all irrigation treatments were renamed. The irrigation regimes for each treatment: low, irrigated twice/hour, 5-8 A.M.; medium, combined treatment of irrigated once/hour and irrigated twice/hour, 2-8 A.M.; and high, irrigated once/hour, 8 P.M.-8 A.M. A, Water-sprayed, 2015; B, water-sprayed, 2016; C, nontreated, nonbagged control, 2015; and D, nontreated, nonbagged control, 2016. 
(Ismail et al. 2016), physiology (Batzer et al. 2010), and fungicide sensitivity (Tarnowski et al. 2003), thereby underlining the potential value of identifying prevalent SBFS taxa in each geographic region (Díaz Arias et al. 2010) for enhancing effectiveness of SBFS management tactics.

The relationship of $P$. gemmifer colony counts on watersprayed apple fruit to cumulative wet hours measured during irrigation periods was considerably stronger than the response to total wetness hours during all periods. We hypothesize that the reason for this difference is that, during the irrigation treatment periods, a higher percentage of LWD hours was caused by precipitation (mainly in the form of spray irrigation) than during other periods. Batzer et al. (2008) documented that, in the absence of overhead irrigation, approximately $70 \%$ of LWD in this same Iowa orchard was caused by dew rather than rain. In the more rain-dominated climate of southeastern Brazil during the fruit maturation period, Spolti et al. (2011) found a significant positive correlation (approximately $90 \%$ ) of SBFS incidence with LWD, rainfall amount per event, and rain intensity. Assuming that precipitation is more effective than dew in transferring water droplets containing budded conidia from one apple to another, the relative prevalence of precipitation over dew during irrigation-treatment periods could explain why colony counts of $P$. gemmifer were more closely correlated with cumulative LWD during the irrigation treatments than with total LWD.

Covering apple fruit with fruit bags from shortly after the firstcover spray until the day of inoculation minimized deposition of external SBFS inoculum. However, the time lag from petal fall until the date the bags were applied ranged from 30 days in 2015 to 12 days in 2016 . Therefore, it is possible that more inoculum from natural sources landed on the fruit in 2015 compared with 2016. Similarly, more SBFS colonies were observed on nontreated fruit that were never bagged than on those that had been bagged before the inoculation date in both years, especially for $P$. gemmifer. However, this difference in timing of bagging seems unlikely to have affected the observed relationships between irrigation treatment and colony counts among the three inoculated SBFS taxa.

A Peltaster sp. was reported to produce conidia through budding in vitro (Johnson et al. 1996). Rapid production of small conidia by means of budding may offer an advantage to Peltaster spp. in dissemination during rainy periods, acting as a reservoir of inoculum for secondary disease cycles via rain splash (Fones et al. 2017). Isolate CUE2b used for inoculation in the present study showed similar identity with MF075291, a sequence from an isolate of a recently described SBFS species, $P$. gemmifer, that displayed the ability to produce secondary conidia in vitro through budding (Rosli et al. 2018). Williamson and Sutton (2000) suggested that the "dripping" appearance of $P$. fructicola colonies on apple fruit resulted from secondary spread by rain splash. The dripping appearance was also observed in the present

Table 4. Least squares means of log-transformed counts of colonies of sooty blotch and flyspeck species on water-sprayed and nontreated apple fruit in 2015 and 2016 in a nonirrigated control plot that received only ambient precipitation

\begin{tabular}{lrrrrr}
\hline & \multicolumn{3}{c}{ Apple fruit treatment and year } \\
\cline { 2 - 3 } \cline { 6 - 7 } Species & \multicolumn{2}{c}{ Water-sprayed } & & \multicolumn{2}{c}{ Nontreated $^{\mathbf{z}}$} \\
\cline { 2 - 3 } \cline { 6 - 7 } & $\mathbf{2 0 1 5}$ & $\mathbf{2 0 1 6}$ & & $\mathbf{2 0 1 5}$ & $\mathbf{2 0 1 6}$ \\
\hline Peltaster gemmifer & $3.13 \mathrm{a}$ & $1.47 \mathrm{a}$ & & $3.58 \mathrm{a}$ & $-0.29 \mathrm{ab}$ \\
Stomiopeltis sp. RS1 & $0.63 \mathrm{~b}$ & $-0.94 \mathrm{~b}$ & & $1.94 \mathrm{~b}$ & $0.28 \mathrm{a}$ \\
Microcyclosporella mali & $-0.41 \mathrm{c}$ & $-0.60 \mathrm{~b}$ & & $1.35 \mathrm{~b}$ & $-1.10 \mathrm{~b}$ \\
\hline
\end{tabular}

$\mathrm{x}$ Means within a column followed by the same letter are not significantly different (least squares means, $P<0.05$ ).

y Apple fruit were bagged shortly after first-cover spray until the day of inoculation.

z Apple fruit were neither bagged nor inoculated, and were selected arbitrarily at harvest. study on fruit inoculated with $P$. gemmifer (Supplementary Fig. S3). M. mali, a predominant SBFS species throughout Europe and the United States (Batzer et al. 2016; Díaz Arias et al. 2010; Ivanović et al. 2010), is also known for abundant yeastlike microcyclic conidiation (Frank et al. 2010). However, rather than the small (approximately $2 \mu \mathrm{m}$ ) single-celled conidia of $P$. gemmifer, M. mali has scolecospores with up to 10 septations, ranging from 15 to $40 \mu \mathrm{m}$ in length (Batzer et al. 2012, 2010; Frank et al. 2010). Thus, differential spore size may have had some effect on dispersal and led to the differential response caused by irrigation.

In contrast, Stomiopeltis spp. have not been found to produce conidia in culture, and only occasionally produce ascospores on overwintered fruit (Abad et al. 2005). Absence of secondary spread in the present study adds to evidence that Stomiopeltis sp. is a monocyclic pathogen. Nevertheless, more than a decade of field research in the Upper Midwest has shown that the ramose SBFS colonies associated with Stomiopeltis spp. are the most abundant and common type of SBFS in this region (Batzer et al. 2005, 2012; Díaz Arias et al. 2010; Ismail et al. 2016; Rosli et al. 2017). Because we did not detect the Stomiopeltis sp. RS1 used during inoculation but, rather, a different Stomiopeltis sp. (RS5), we conclude that the inoculum source for ramose mycelial types of SBFS originated from ambient, natural sources. Ismail et al. (2016) showed that most of the fruit inoculations by Stomiopeltis sp. RS1 and RS2 and M. mali occurred during the first 2 weeks after fruit set, whereas new inoculations later in the season were relatively few. In contrast, initiation of new Peltaster spp. colonies remained relatively constant throughout a growing season with average rainfall and sharply increased during a year with above-average rainfall (Ismail et al. 2016). Our study's findings support these observations, which appear to suggest that infection of apple fruit by Stomiopeltis spp. is monocyclic whereas infection by Peltaster spp. is polycyclic.

Another SBFS pathogen, Zygophiala sp., produced conidiophores and conidia in vitro and on the apple surface (Gao et al. 2014; Williamson and Sutton 2000). Gao et al. (2014) also reported secondary production of Zygophiala sp. conidia on apple fruit; conidia germinated on the apple surface and produced sclerotium-like bodies (clustered and thickened hyphae) containing secondary conidia.

Although the present study did not measure the spatial extent or rate of dissemination of SBFS taxa in the field, counting colonies on nontreated apple fruit provided convincing indirect evidence of dissemination. The present study provides a foundation for quantitative studies to clarify relationships between environmental wetness and SBFS dissemination, and to discern differences among SBFS taxa in dispersion dynamics. In order to assess spatial dissemination

Table 5. Cumulative ambient rainfall and leaf wetness duration (LWD) for irrigation treatments at Iowa State University Horticulture Research Station in 2015 and 2016

\begin{tabular}{lcr}
\hline & \multicolumn{2}{c}{ Year } \\
\cline { 2 - 3 } Rainfall and wetness duration & $\mathbf{2 0 1 5}$ & $\mathbf{2 0 1 6}$ \\
\hline Cumulative rainfall (mm) & 394.5 & 503.9 \\
Cumulative LWD (hours) & & \\
1 & 904.4 & 912.1 \\
2 & 929.6 & 957.7 \\
3 & 941.1 & 856.9 \\
4 & $1,042.9$ & $1,146.9$ \\
5 & 827.1 & 771.4 \\
\hline y & \multicolumn{2}{c}{ Data collected at the ISU Horticulture Research Station, Gilbert, Iowa, at a } \\
weather station located approximately 1,000 m from the experimental or- \\
chard, from date of inoculation to date of harvest (https://www.mesonet. \\
agron.iastate.edu/). \\
z Cumulative LWD for each irrigation treatment from date of inoculation to \\
date of harvest. Irrigation regimes for each treatment: 1 = irrigated twice/ \\
hour, 5-8 A.M., 2 = irrigated once/hour, 2-8 A.M., 3 = irrigated twice/hour, \\
2-8 A.M., 4 = irrigated once/hour, 8 P.M.-8 A.M., and 5 = no irrigation.
\end{tabular}



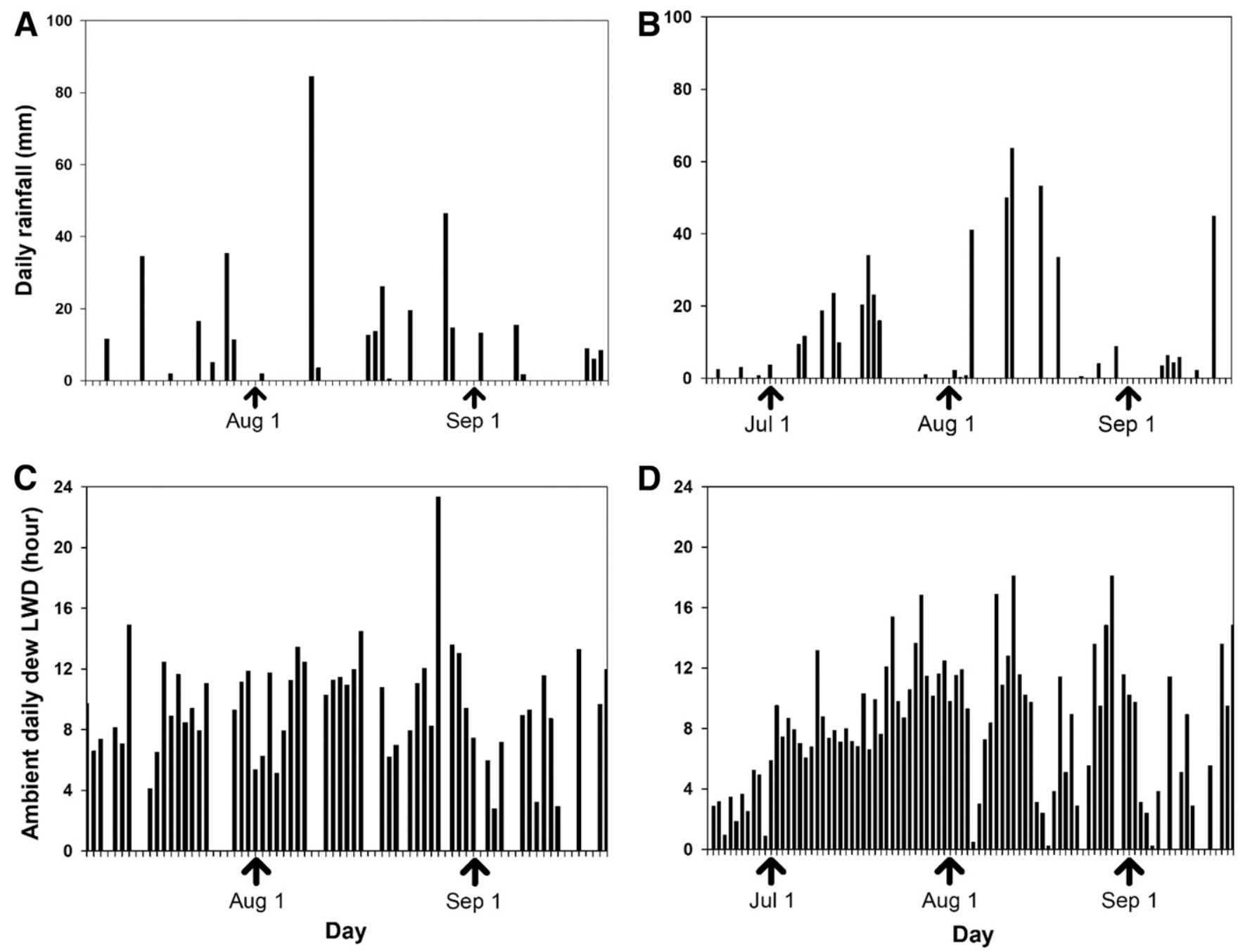

Fig. 3. Daily weather data (total rainfall and dew) from day of inoculation to harvest at lowa State University Horticulture Research Station. Rainfall data were collected from a weather station located 1,000 m from the experimental orchard (https://www.mesonet.agron.iastate.edu/), and daily dew data were obtained from sensors located in the nonirrigated control plot of the experimental orchard. LWD = leaf wetness duration. A and C, 2015; and B and D, 2016.

on a larger spatial scale and verify the production of secondary inoculum in the field, a logical further step would be to monitor conidia of SBFS taxa using spore traps at different time intervals after inoculation.

\section{Acknowledgments}

We thank N. Howell and L. Schroeder at the Iowa State University Horticulture Research Station for their invaluable assistance on this project; and the summer crews from the Gleason laboratory for their help in the field.

\section{Literature Cited}

Abad, G., Raleigh, N. C., and Sutton, T. B. 2005. Morphological and molecular identification of Stomiopeltis uniloculata sp. nov. and Stomiopeltis multiloculata sp. nov. associated with apple fruit in North Carolina. Phytopathology 95:960.

Batzer, J. C., Gleason, M. L., Harrington, T. C., and Tiffany, L. H. 2005. Expansion of the sooty blotch and flyspeck complex on apples based on analysis of ribosomal DNA gene sequences and morphology. Mycologia 97: 1268-1286.

Batzer, J. C., Gleason, M. L., Taylor, S. E., Koehler, K. J., and Monteiro, J. E. B. M. 2008. Spatial heterogeneity of leaf wetness duration in apple trees and its influence on performance of a warning system for sooty blotch and flyspeck. Plant Dis. 92:164-170.

Batzer, J. C., Hernandez Rincon, S., Mueller, D. S., Petersen, B. J., Le Corronc, F., McManus, P. S., Dixon, P. M., and Gleason, M. 2010. Effect of temperature and nutrient concentration on the growth of six species of sooty blotch and flyspeck fungi. Phytopathol. Mediterr. 49:3-10.

Batzer, J. C., Sisson, A. J., Harrington, T. C., Mayfield, D. A., and Gleason, M. L. 2012. Temporal patterns in appearance of sooty blotch and flyspeck fungi on apples. Microb. Ecol. 64:928-941.
Batzer, J. C., Stensvand, A., Mayfield, D. A., and Gleason, M. L. 2015. Composition of the sooty blotch and flyspeck complex on apple in Norway is influenced by location and management practices. Eur. J. Plant Pathol. 141: 361-374.

Batzer, J. C., Weber, R. W., Mayfield, D. A., and Gleason, M. L. 2016. Diversity of the sooty blotch and flyspeck complex on apple in Germany. Mycol. Prog. 15:2.

Brown, E. M., and Sutton, T. B. 1995. An empirical model for predicting the first symptoms of sooty blotch and flyspeck of apples. Plant Dis. 79:1165-1168.

Díaz Arias, M. M., Batzer, J. C., Harrington, T. C., Wang Wong, A., Bost, S. C., Cooley, D. R., Ellis, M. A., Hartman, J. R., Rosenberger, D. A., Sundin, G. W., Sutton, T. B., Travis, J. W., Wheeler, M. J., Yoder, K. S., and Gleason, M. L. 2010. Diversity and biogeography of sooty blotch and flyspeck fungi on apple in the eastern and midwestern United States. Phytopathology 100:345-355.

Duttweiler, K. B., Sun, G. Y., Batzer, J. C., Harrington, T. C., and Gleason, M. L. 2008. An RFLP-based technique for identifying fungi in the sooty blotch and flyspeck complex on apple. Plant Dis. 92:794-799.

Fones, H. N., Eyles, C. J., Kay, W., Cowper, J., and Gurr, S. J. 2017. A role for random, humidity-dependent epiphytic growth prior to invasion of wheat by Zymoseptoria tritici. Fungal Genet. Biol. 106:51-60.

Frank, J., Crous, P. W., Groenwald, J. Z., Oetrel, B., Hyde, K. D., Phengsintham, P., and Shroers, H. J. 2010. Microcyclospora and Microcyclosporella: Novel genera accommodating epiphytic fungi causing sooty blotch on apple. Persoonia 24:93-105.

Gao, L., Ma, Y., Zhao, W., Wei, Z., Gleason, M. L., Chen, H., Hao, L., Sun, G., and Zhang, R. 2015. Three new species of Cyphellophora (Chaetothyriales) associated with sooty blotch and flyspeck. PLoS One 10:e0136857.

Gao, L., Sun, G., Zhang, R., and Gleason, M. L. 2014. Secondary spread of Zygophiala wisconsinensis on the surface of apple fruit. Eur. J. Plant Pathol. 139:117-124.

Gillespie, T. J., and Kidd, G. E. 1978. Sensing duration of leaf moisture retention using electrical impedance grids. Can. J. Plant Sci. 58:179-187. 
Gleason, M. L., Batzer, J. C., Sun, G., Zhang, R., Arias, M. M. D., Sutton, T. B., Crous, P. W., Ivanović, M., McManus, P. S., Cooley, D. R., and Mayr, U. 2011. A new view of sooty blotch and flyspeck. Plant Dis. 95:368-383.

Gleason, M. L., Zhang, R., Batzer, J. C., and Sun, G. 2019. Stealth pathogens: The sooty blotch and flyspeck fungal complex. Annu. Rev. Phytopathol. 57: 135-164.

Hall, T. A. 1999. BioEdit: A user-friendly biological sequence alignment editor and analysis program for Windows 95/98/NT. Nucleic Acid Symp. Ser. 41: 95-98

Ismail, S. I., Batzer, J. C., Harrington, T. C., and Gleason, M. L. 2016. Phenology of infection on apple fruit by sooty blotch and flyspeck species in Iowa apple orchards. Plant Dis. 100:352-359.

Ivanović, M. M., Ivanović, M. S., Batzer, J. C., Tatalović, N., Oertel, B., Latinović, J., Latinović, N., and Gleason, M. L. 2010. Fungi in the apple sooty blotch and flyspeck complex from Serbia and Montenegro. J. Plant Pathol. 92:65-72.

Johnson, E. M., and Sutton, T. B. 2000. Response of two fungi in the apple sooty blotch complex to temperature and relative humidity. Phytopathology 90: 362-367.

Johnson, E. M., Sutton, T. B., and Hodges, C. S. 1996. Peltaster fructicola: A new species in the complex of fungi causing apple sooty blotch disease. Mycologia 88:114-120.
Johnson, E. M., Sutton, T. B., and Hodges, C. S. 1997. Etiology of apple sooty blotch disease in North Carolina. Phytopathology 87:88-95.

Medjedović, A., Frank, J., Schroers, H. J., Oertel, B., and Batzer, J. C. 2014 Peltaster cerophilus is a new species of the apple sooty blotch complex from Europe. Mycologia 106:525-536.

Rosli, H., Batzer, J. C., Harrington, T. C., and Gleason, M. L. 2018. Peltaster gemmifer: A new species in the sooty blotch and flyspeck species complex from the United States. Mycologia 110:822-834.

Rosli, H., Mayfield, D. A., Batzer, J. C., Dixon, P. M., Zhang, W., and Gleason, M. L. 2017. Evaluating the performance of a relative humidity-based warning system for sooty blotch and flyspeck in Iowa. Plant Dis. 101:1721-1728.

Spolti, P., Valdebenito-Sanhueza, R. M., Gleason, M. L., and Del Ponte, E. M. 2011. Inoculum and infection dynamics of the sooty blotch and flyspeck complex of apples in southern Brazil. J. Plant Pathol. 93:497-501.

Tarnowski, T., Batzer, J., Gleason, M. L., Helland, S., and Dixon, P. 2003. Sensitivity of newly identified clades in the sooty blotch and flyspeck complex on apple to thiophanate-methyl and ziram. Plant Health Prog. 4.

Williamson, S. M., and Sutton, T. B. 2000. Sooty blotch and flyspeck of apple: Etiology, biology, and control. Plant Dis. 84:714-724.

Zhao, W., Hou, Y., Bai, J., Zhang, W., Gao, L., Zhang, R., Gleason, M. L., and Sun, G. 2016. A new species of Zasmidium associated with sooty blotch and flyspeck. Phytotaxa 258:190-194. 